\title{
Глубокие центры радиационных дефектов в монокристаллах CdZnTe, созданные потоком быстрых нейтронов
}

\author{
(C) С.В. Пляцко, Л.В. Рашковецкий
}

Институт фризики полупроводников им. В.Е. Лашкарева Национальной академии наук Украины, 03028 Киев, Украина

E-mail: plyatsko@isp.kiev.ua; rashlv@ukr.net

(Получена 30 августа 2017 г. Принята к печати 5 сентября 2017 г.)

Исследовано влияние потока быстрых нейтронов $\left(\Phi=10^{14}-10^{15} \mathrm{~cm}^{-2}\right)$ на электрофизические и фотолюминесцентные свойства монокристаллов $p$-CdZnTe. Проведен изотермический отжиг $(T=400-500 \mathrm{~K})$ и определена энергия активации диссоциации радиационных дефектов, которая оказалась равной $E_{D} \approx 0.75$ эВ.

DOI: 10.21883/FTP.2018.03.45615.8373

\section{1. Введение}

Теллурид кадмия - широкозонное II-VI соединение, которое благодаря высокому среднему атомному номеру и хорошему произведению подвижности на время жизни носителей как для электронов, так и дырок широко используется для $X-\gamma$ - и ядерных детекторов $[1,2]$. Монокристаллы теллурида кадмия благодаря своему высокому удельному сопротивлению и широкой запрещенной зоне имеют низкие токи утечки, вследствие чего детекторы на их основе могут функционировать при комнатной температуре. Кроме того, большая плотность и среднее значение атомного номера предполагают высокое поглощение в области энергий 10-500 кэВ, что делает их конкурентоспособными с Ge-детекторами и сцинтилляторами. $\mathrm{CdTe}: \mathrm{Cl}$, как полагают, является одним из лучших полупроводниковых материалов для ядерного детектирования, но производство больших слитков с высоким удельным сопротивлением и с высоким произведением подвижности на время жизни носителей изделия все еще затруднено. Хорошей альтернативой является $\mathrm{CdZnTe}$, поскольку его удельное сопротивление на порядок выше, чем $\mathrm{CdTe}: \mathrm{Cl}$, он более термостабилен и может быть выращен большего размера при уменьшенной стоимости. Тем не менее использование $\mathrm{CdTe}$ и $\mathrm{CdZnTe}$ в качестве ядерных датчиков все еще испытывает недостаток в полной характеристике их поведения при различных эксплуатационных условиях. Существует незначительное количество экспериментальных работ по взаимодействию потока тепловых и быстрых нейтронов с детекторами CdTe [3] и $\mathrm{CdZnTe} \mathrm{[4].} \mathrm{Было} \mathrm{установлено,} \mathrm{что} \mathrm{в} \mathrm{электронном} \mathrm{типе}$ проводимости поток нейтронов создает значительную концентрацию рассеивающих центров, что приводит к значительному понижению подвижности и уменьшению времени жизни электронов с увеличением флюэнса нейтронов. В p-CdZnTe при такой же концентрации радиационных дефектов таких эффектов не обнаружено. Кроме того, из PICTS (спектроскопия фотоиндуцированного нестационарного тока) исследований следует, что независимо от типа применяемого облучения $(\gamma$-, низко- и высокоэнергетические нейтроны и электроны) возникают дефекты в одном и том же энергетическом интервале, но с разной концентрацией.

Также были получены результаты, подтверждающие деградацию энергетического разрешения, сдвига фотопика и ухудшения собирающей эффективности заряда при радиационном повреждении [3] и уменьшение времени нарастания сигнала и возрастание чувствительности [4] в нейтронно-облученных детекторах. Стабильность же радиационных дефектов, которые возникают при нейтронном облучении, не исследовалась. Именно по этой причине в представленной работе проведено изучение влияния быстрых нейтронов на свойства монокристаллов CdZnTe. Основное внимание уделено спектру возникающих радиационных дефектов в зависимости от дозы облучения и их стабильности во времени при разных температурах.

\section{2. Эксперимент}

Для исследований были подготовлены монокристаллические пластины $\mathrm{Cd}_{1-x} \mathrm{Zn}_{x} \mathrm{Te}$ ориентации (111), которые были получены со слитков, выращенных вертикальным методом Бриджмена, с удельным сопротивлением $\rho_{300}=4 \cdot 10^{3} \mathrm{OM} \cdot \mathrm{cm}$ и величиной оптического пропускания $\tau_{10 \text { мкм }}=0.5$. Все исследуемые образцы $p-\mathrm{Cd}_{1-x} \mathrm{Zn}_{x}$ Te $(x<0.04)$ облучались быстрыми нейтронами $\left(1 \cdot 10^{14}, 5 \cdot 10^{14}\right.$ и $\left.1 \cdot 10^{15} \mathrm{~cm}^{-2}\right)$ в реакторе ВВР с эффективной энергией 1 МэВ. Температура образцов в процессе облучения не превышала $25^{\circ} \mathrm{C}$. После облучения образцы выдерживали определенное время в специальных боксах и затем проводились измерения их параметров. Омические контакты к образцам формировали химическим осаждением золота. Для исследования спектров низкотемпературной фотолюминесценции (НФЛ) образцы до и после облучения раскалывали по плоскости спайности (110). Спектры НФЛ образцов исследовались при температуре жидкого гелия $(4.2 \mathrm{~K})$. Возбуждение осуществлялось аргоновым или $\mathrm{He}-\mathrm{Ne}$ лазерами на длине волны $\lambda=488.0$ и $\lambda=632.0$ нм соответственно. Излучение анализировалось решеточным монохроматором МДР-23. 


\section{3. Электрофизические свойства}

Кристаллам $\mathrm{Cd}_{1-x} \mathrm{Zn}_{x} \mathrm{Te}(x<0.04)$ характерны низкие значения энергии образования собственных дефектов и комплексов в присутствии фоновых примесей, энергетические уровни которых расположены в запрещенной зоне $E_{v}=(0.06-0.22)$ эВ. В зависимости от условий получения и степени очистки, плотность состояний на уровнях будет определять проводимость и оптические свойства кристаллов. Идентификация уровней представляется трудной задачей, поэтому зачастую приписывается собственным дефектам, остаточным неконтролируемым примесям или их комплексам. Для компенсации в монокристаллах $p$-CdZnTe акцепторного действия собственных дефектов можно использовать либо отжиг в парах собственных компонентов, либо легирование, либо лазерное облучение $\left(\hbar \omega \ll E_{g}\right)$ [5-11], либо радиационное облучение. Взаимодействие кристаллов $p-\mathrm{Cd}_{1-x} \mathrm{Zn}_{x} \mathrm{Te}$ с потоком быстрых нейтронов приводит к увеличению удельного сопротивления $\rho$ с дозой облучения $\Phi$. Зависимость $\rho(\Phi)$ имеет разный характер при низкой и комнатной температурах. В отличие от $\mathrm{Si}$ и $\mathrm{GaAs} \rho(\Phi)$ при низких значениях флюенса, удельному сопротивлению характерна область быстрого увеличения. С ростом флюенса изменение $\rho$ при низких температурах замедляется (рис. 1 , кривая $T=100 \mathrm{~K}$ ), а при комнатной температуре проходит через максимум и опускается до значений, соответствующих начальной дозе облучения (рис. 1 , кривая $T=300 \mathrm{~K}$ ). Полученные зависимости отображают сложный процесс дефектообразования в монокристаллах $\mathrm{Cd}_{1-x} \mathrm{Zn}_{x} \mathrm{Te}$ потоком быстрых нейтронов и конкурирующий их вклад в проводимость с ростом $\Phi$.

В совершенных монокристаллах $\mathrm{Cd}_{1-x} \mathrm{Zn}_{x} \mathrm{Te}$, в области малых значений $x$ доминирующим механизмом рассеяния носителей в области $T>100 \mathrm{~K}$ является рассеяние на оптических фононах, поэтому подвижность от температуры изменяется пропорционально $T^{-3 / 2}$.

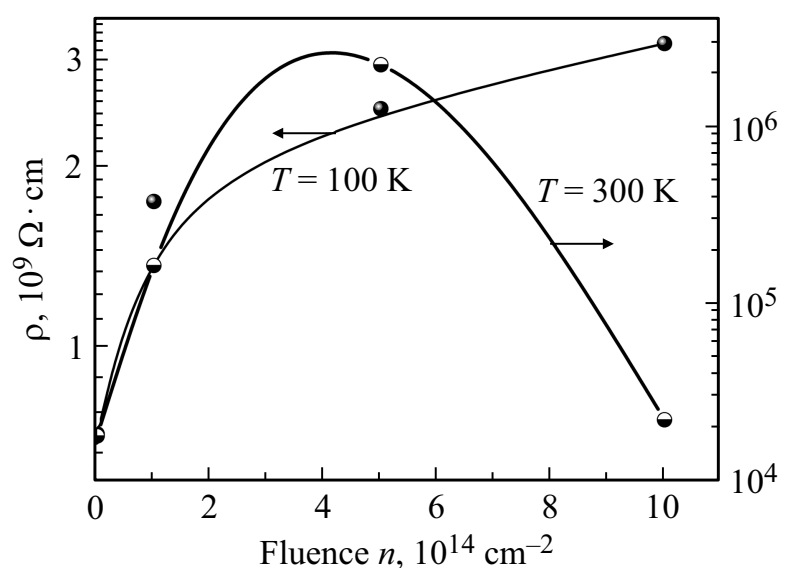

Рис. 1. Зависимость удельного сопротивления монокристаллов $p-\mathrm{Cd}_{1-x} \mathrm{Zn}_{x}$ Те от флюенса быстрых нейтронов с $E_{n}=1 \mathrm{MэВ}$ при начальном значении $\rho_{300}=1.80 \cdot 10^{4} \mathrm{OM} \cdot \mathrm{cm}$ (кривая $T=300 \mathrm{~K})$ и $\rho_{100}=7.17 \cdot 10^{8} \mathrm{OM} \cdot$ см (кривая $\left.T=100 \mathrm{~K}\right)$.

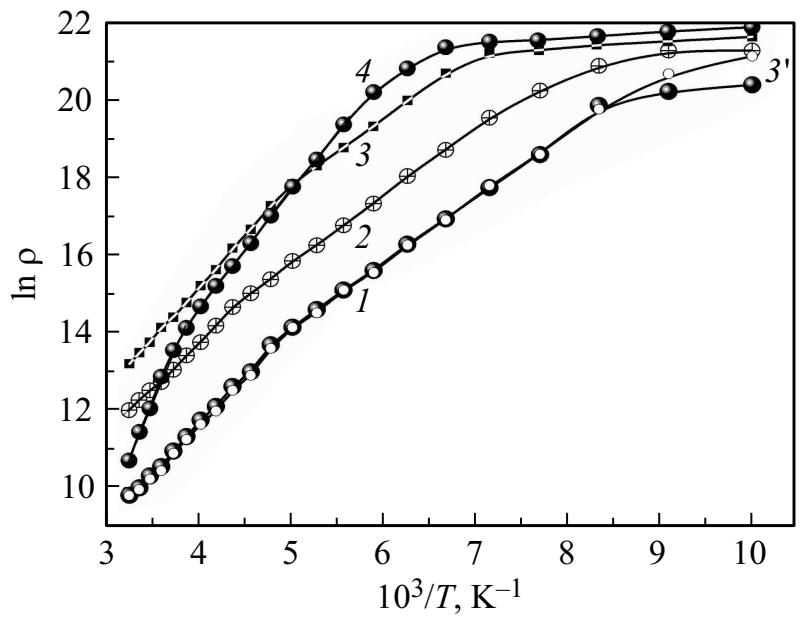

Рис. 2. Температурные зависимости удельного сопротивления монокристаллов $p$-CdZnTe. 1 - до облучения быстрыми нейтронами $\Phi=0,2-\Phi=1 \cdot 10^{14} \mathrm{~cm}^{-2}, 3-5 \cdot 10^{14} \mathrm{~cm}^{-2}$, $4-1 \cdot 10^{15} \mathrm{~cm}^{-2} \cdot 3^{\prime}-$ после изотермического отжига при $T=400 \mathrm{~K}$.

Эффективные плотности состояний в свою очередь в зоне проводимости $N_{c}=2\left(m_{n} k T / 2 \pi \hbar\right)^{3 / 2}$ и валентной зоне $N_{v}=2\left(m_{p} k T / 2 \pi \hbar\right)^{3 / 2}$ пропорциональны $T^{3 / 2}$ $\left(m_{n}\right.$ и $m_{p}$ - эффективная масса электрона и дырки). Тогда температурная зависимость удельного сопротивления $\rho=1 /\left(e n \mu_{n},+e p \mu_{p}\right)-$ это экспоненциальная функция температуры $\left(\mu_{n}\right.$ и $\mu_{p}-$ подвижности электронов и дырок), и зависимости $\rho(T)$ в координатах $\ln (\rho)$ от $1000 / T$, представленные прямыми линиями, можно использовать для определения энергии активации проводимости материала $\Delta E$.

На рис. 2 приведены зависимости удельного сопротивления от обратной температуры $\rho(1 / T)$ для кристаллов с разной дозой облучения. По изменению наклона видно, что на фоне изменения удельного сопротивления энергетический спектр дефектов изменяется.

До начала облучения в зависимости $\rho(1 / T)$ в кристаллах наблюдается один низкотемпературный наклон $E_{1 a}$, которому соответствует энергия активации $E_{1 a} \approx 0.135$ эВ. В области температур $T>200 \mathrm{~K}$ наблюдается переход к высокотемпературному наклону более глубоких дефектов $E_{2 a}=E_{v}+0.21$ эВ. Несмотря на то что кристаллы получены из шихты с чистотой $6 N$, нельзя однозначно утверждать, что относительно мелкий уровень $E_{1 a} \approx E_{v}+0.135$ эВ в исходном кристалле принадлежит собственным дефектам, например вакансиям $\mathrm{Cd}$. Плотность состояний на уровне не превышает $5 \cdot 10^{13} \mathrm{~cm}^{-3}$, что ниже концентрации фоновых примесей.

В облученных кристаллах ход зависимостей $\rho(1 / T)$ качественно не изменяется для флюенса быстрых нейтронов $\Phi=(1-5) \cdot 10^{14} \mathrm{~cm}^{-2}$ во всей области температур.

При этом проявляется уменьшение плотности состояний на уровне $E_{1 a}$ и $E_{2 a}$. Плотность состояний на уровне $E_{1 a}$ и $E_{2 a}$ падает более чем на 2 порядка и не превышает 
$5 \cdot 10^{10} \mathrm{~cm}^{-3}$. Уменьшение концентрации носителей может быть также связано с вкладом в проводимость более глубоких уровней, плотность состояний на которых возрастает при увеличении флюенса быстрых нейтронов. Для флюенса $\Phi=1 \cdot 10^{15} \mathrm{~cm}^{-2}$ совершенно четко видно, что на зависимостях $\rho(1 / T)$ проявляются также два наклона $E_{2 a}=E_{v}+0.35$ эВ и $E_{4 a}=E_{v}+0.51$ эВ. Облучение $n-\mathrm{CdZnTe}$ быстрыми нейтронами приводит также к серии радиационных дефектов с уровнями в запрещенной зоне разной плотности состояний $-E_{c}-0.5$, $E_{c}-0.02, E_{c}-0.7, E_{c}-0.43$ эВ [5-8], однако при более низких потоках нейтронов.

Радиационные дефекты, возникающие в кристалле после облучения быстрыми нейтронами, при температурах, близких к комнатной, не проявляли тенденции к релаксации на протяжении нескольких месяцев. Однако было замечено, что при повышении температуры выше комнатной происходит уменьшение удельного сопротивления со временем. Скорость релаксации увеличивалась с повышением температуры $T_{\text {rel. }}$ После изотермического отжига $400 \leq T \leq 450 \mathrm{~K}$ на зависимостях $\rho(1 / T)$ проявились два наклона (рис. 2, кривая $3^{\prime}$ ), соответствующие энергии активации $E_{1 a}=E_{v}+0.135$ эВ и $E_{2 a}=E_{v}+0.21$ эВ. Значение удельного сопротивления при этом во всей области температур уменьшалось почти до исходного в необлученных кристаллах. Повышение температуры отжига выше $T \geq 450 \mathrm{~K}$ приводило в некоторых кристаллах к более глубокому падению $\rho$ и проявлению в $\rho(1 / T)$ только уровня $E_{1 a}=E_{v}+0.135$ эВ, что говорит об увеличении плотности состояний на $E_{1 a}$ в результате диссоциации более глубоких центров, а также о его определяющем вкладе в проводимость в температурном интервале $77 \leq T \leq 320 \mathrm{~K}$.

Из зависимости $p(t)$ концентрации дырок $p$ в $p$-CdZnTe от времени изотермического отжига $t$ (рис. 3 ), полученной для температур $T_{1}=350$ и $T_{2}=400 \mathrm{~K}$,

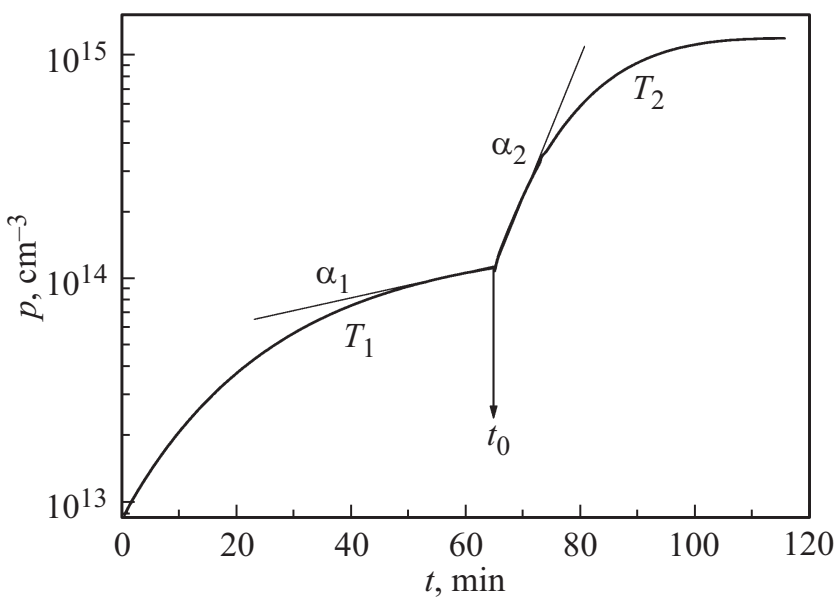

Рис. 3. Зависимость концентрации дырок $p$ в облученных быстрыми нейтронами $\left(\Phi=1 \cdot 10^{15} \mathrm{~cm}^{-2}\right)$ монокристаллах $p$-CdZnTe от времени $t$ изотермического отжига $\left(T_{1}=350\right.$, $\left.T_{2}=400 \mathrm{~K}\right)$. можно определить энергию активации $E_{D}$ изотермического отжига радиационных дефектов, поскольку наклоны $\alpha_{1}$ и $\alpha_{2}$ кривой $p(t)$ в момент времени $t_{0}$ перехода от температуры отжига $T_{1}$ к $T_{2}$ связаны с энергией активации $E_{D}$ следующим образом [12]: $E_{D}=k\left(T_{1} T_{2} /\left(T_{1}-T_{2}\right) \ln \left(\alpha_{1} / \alpha_{2}\right)\right)$. Рассчитанная величина энергии активации оказалась равной $E_{D} \approx 0.75$ эВ.

Уменьшение удельного сопротивления при температуре отжига $T \geq 450 \mathrm{~K}$ говорит о том, что в некоторых кристаллах существуют также более устойчивые радиационные дефекты с энергией активации выше $E_{D}$.

\section{4. Экситонная и $D A$ области спектра НФЛ}

Обычно в нелегированных монокристаллах $p$-CdTe и $p$-CdZnTe c малым содержанием цинка в спектрах краевой НФЛ наблюдаются линия излучения связанного экситона на нейтральном акцепторе $A^{0} X$ и иногда очень слабые полосы, обусловленные излучением свободного экситона и экситона, связанного на нейтральном доноре $D^{0} X[13]$. Связанный экситон $D^{0} X$ в специально не легированных кристаллах проявляется слабо [14], но с введением $\mathrm{Zn}$ интенсивность $D^{0} X$-полосы становится более заметной [15]. Исходные кристаллы, как уже говорилось выше, имели относительно низкое значение удельного сопротивления и дырочный тип проводимости с соответствующим спектром краевой НФЛ (рис. 4, кривая 0 ). Спектр НФЛ состоял из слабой линии $A^{0} X_{1}-1.5912$ эВ, интенсивной линии $A^{0} X_{2}-1.5851$ эВ, ее фононного повторения $\left(A^{0} X_{2}-\mathrm{LO}\right)-1.5636$ эВ $\left(\hbar \omega_{\mathrm{LO}}=0.0215\right.$ эВ $)$ и довольно широкой бесструктурной полосы $D A-$ 1.5447 эВ. Интенсивная линия $D A$-излучения указывает на значительную концентрацию мелких акцепторных центров.

Доминирующей в экситонной области спектра является $A^{0} X_{2}$-линия. В низкоэнергетической спектральной области за экситонной областью наблюдается широкая полоса 1.5447 эВ со своим фононным повторением 1.5232 эВ, которая связана с переходами зона проводимости-нейтральный акцептор $(e A)$ и донорноакцепторная эмиссия $D A$ (рис. 4).

В процессе облучения с увеличением флюенса во всех монокристаллах наблюдается зависимость интенсивности полос $A^{0} X_{1}$ и $A^{0} X_{2}$ от дозы облучения (рис. 5). Зависимость имеет нелинейный характер как для $A^{0} X_{1}$, так и для $A^{0} X_{2}$. Незначительный спад интенсивности при малых потоках быстрых нейтронов свидетельствует о том, что в монокристалах присутствуют неустойчивые дефекты, концентрация которых уменьшается при облучении. Дальнейший рост интенсивности экситонних полос с флюенсом указывает на рост мелких нейтральных акцепторов, на которых происходит аннигиляция экситонов.

Для $A^{0} X_{2}$ характерным является наличие максимума (при $\Phi=5 \cdot 10^{14} \mathrm{~cm}^{-2}$ ) с дальнейшим спадом интенсивности, что объясняется процессами безызлучательной рекомбинации. В процессе облучения с увеличением 


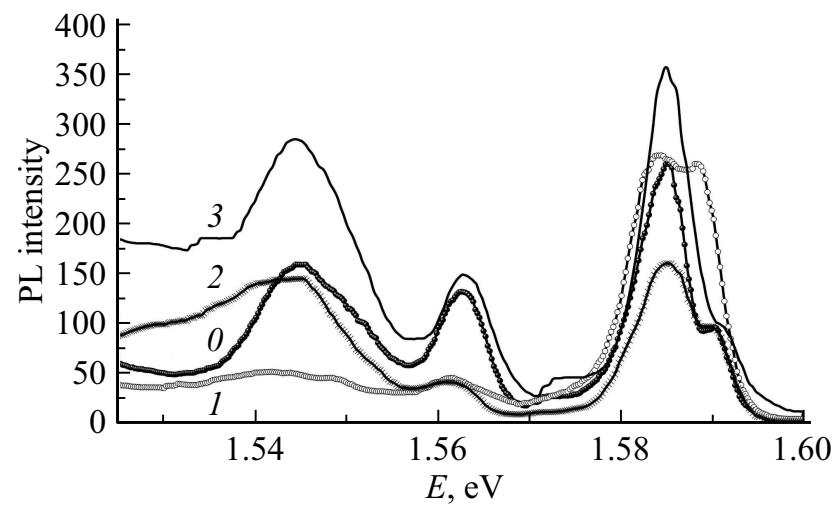

Pис. 4. Экситонная и $e A+D A$ области спектра НФЛ. Цифры возле кривых - флюенс облучения в $\mathrm{cm}^{-2} .0-$ до облучения, $1-1 \cdot 10^{14}, 2-5 \cdot 10^{14}, 3-1 \cdot 10^{15}$.

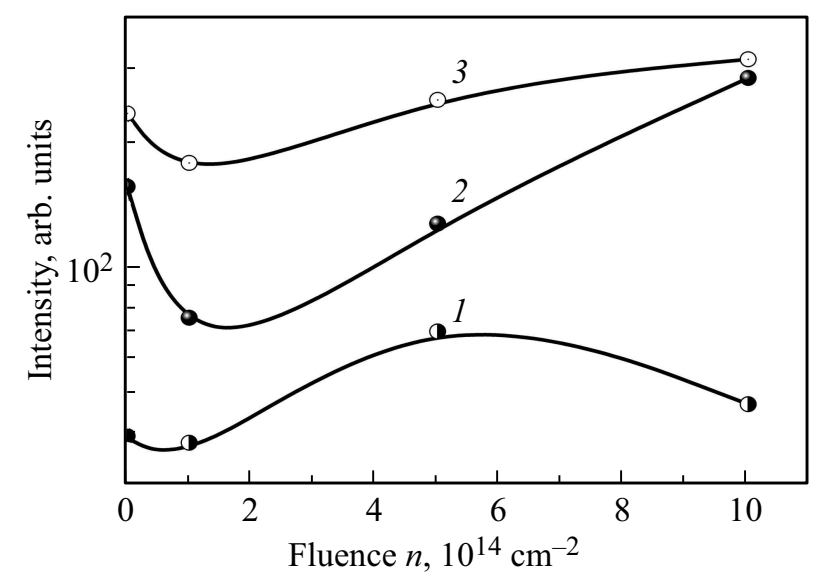

Рис. 5. Зависимость интенсивности НФЛ полос $A^{0} X_{1}$ (кривая 1), $e A+D A$ (кривая 2) и $A^{0} X_{2}$ (кривая 3) от флюенса быстрых нейтронов.

флюенса энергетическое положение $A^{0} X_{1}$ и $A^{0} X_{2}$ обычно не изменяется, но в некоторых кристаллах такое смещение все же наблюдалось за счет радиационностимулированных механических напряжений в окрестности нейтрального акцептора. Но как в первом, так и во втором случае наблюдалось изменение интенсивности экситонных полос $A^{0} X_{1}$ и $A^{0} X_{2}$ и полос $e A+D A$ (рис. 5).

В области излучения $e A+D A$ с увеличением флюенса изменение интегральной интенсивности линии происходит не монотонно - при малых потоках $\left(\Phi=1 \cdot 10^{14} \mathrm{~cm}^{-2}\right)$ интенсивность уменьшается более чем в 3 раза, затем возрастает более чем в 2 раза относительно исходного кристалла. Положение полосы $e A+D A$ при этом не изменяется.

Правило Хайнеса [16] в СdTe не выполняется в полной мере - энергия связи экситона на нейтральном доноре составляет $\sim 0.25 E_{d}$, а для энергии связи экситона на нейтральном акцепторе характерен большой разброс по энергии в пределах от 0.046 до $0.13 E_{a}$, что не дает возможности оценить энергии активации $E_{a}$ из известных энергий связи для экситонов на нейтральном акцепторе. Поэтому энергия активации $E_{a}$ может быть определена из энергетического положения пика $e A+D A$-излучения как

$$
E_{\mathrm{PL}}^{e A^{0}}=E_{g}-E_{a}+\frac{1}{2} k T_{e} .
$$

Для низких уровней возбуждения температуру электронов $T_{e}$ можно полагать равной температуре решетки. Реально эта спектральная область всегда является проблематичной, поскольку полосы ФЛ сильно перекрываются, а в кристаллах $\mathrm{CdZnTe}$ с содержанием цинка выше $x \geq 0.01$ эти полосы практически никогда спектрально не разрешаются. После облучения $e A+D A$ полоса 1.5423 эВ со своими фононными повторениями 1.5211 эВ в этой области спектра становится доминирующей.

По положению пиков $e A+D A$, полагая форму линии гауссовой, была определена энергия активации акцепторов -60.9 мэВ.

\section{$A$-центры}

В твердом растворе CdZnTe спектральная область 1.3-1.5эВ практически всегда присутствует в НФЛ со значительным распределением интенсивности по слитку. Природа полосы люминесценции $A$-центров дискутируется до настоящего времени, но практически все экспериментальные результаты сводятся к тому, что по своей природе $A$-центры являются комплексами, включающими вакансии кадмия (одну или более) и донорный центр, который не является примесью замещения в металлической подрешетке.

В исходных кристаллах в спектрах НФЛ наблюдалась $D-A$-область в спектральном интервале $1.3-1.5$ эВ с LO фононной структурой. Интенсивность фононных повторений можно описать распределением Пуассона:

$$
I_{n}(E)=I_{0} \sum_{n} \frac{\exp (-S) S^{n}}{n} \delta\left(E_{0}-n \hbar \omega-E\right),
$$

где $S$ - фактор Хуанга-Риса, который характеризирует величину электрон-фононного взаимодействия, $I_{0}-$

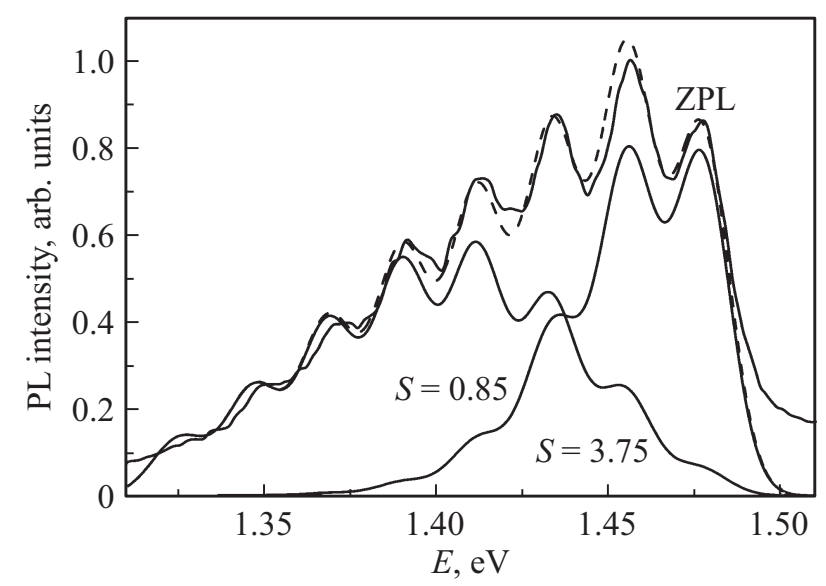

Рис. 6. Нормированные спектры $A$-центров. Штриховая линия - результат аппроксимации на основе формулы (2). На рисунках символом ZPL обозначено положение бесфононных линий. 
интенсивность бесфононной линии. Общая полоса излучения может быть аппроксимирована распределением Пуассона с гауссовыми линиями для каждого отдельного максимума. В результате такой процедуры аппроксимации наилучшее совпадение с экспериментом получалось для суперпозиции бесфононных линий (ZPL) при $S=3.75$ и 0.85 и при положении $h v_{\mathrm{ZPL} 1}=1.4760$ и 1.4770 эВ соответственно (рис. 6).

Глубина залегания $A$-центра определена из спектрального положения бесфононных полос, используя выражение для излучения донорно-акцепторных пар $\left(h v=E_{g}-\left(E_{a}+E_{d}\right), E_{g}=1.606\right.$ эВ и $E_{d}=0.0138$ эВ), в предположении, что переходы происходят с мелкого донора $\left(E_{d}=13.8\right.$ мэВ), положение которого, как следует из оценок в приближении эффективной массы, не зависит от химической природы примеси. Энергия ионизации акцепторов для $h v_{\mathrm{ZPL}}=1.4760$ и 14770 эВ составляет $E_{1 a}=0.116$ и $E_{2 a}=0.115$ эВ соответственно.

Значение энергий активации акцепторных уровней $E_{a}$ в запрещенной зоне попадает в тот интервал энергий, который обычно приписывают сложным дефектам, в состав которых входят собственные дефекты, фоновые примеси или примеси специально введенные.

\section{5. Заключение}

Полученные результаты свидетельствуют о том, что в монокристаллах $p$-CdZnTe при облучении быстрыми реакторными нейтронами наблюдается зависимость интенсивности рекомбинационного излучения в спектральной области 1.3-1.6 эВ от флюенса. Новые рекомбинационные центры в этой области при этом не проявляются. Из исследований электрофизических свойств следует, что поток быстрых нейтронов $\Phi=1 \cdot 10^{15} \mathrm{~cm}^{-2}$, взаимодействуя с собственными точечными дефектами и их комплексами, приводит к образованию в запрещенной зоне глубоких уровней $\left(E_{3 a}=E_{v}+0.35\right.$ эВ, $E_{4 a}=E_{v}+0.51$ эВ), которые, как и в $n$-CdZnTe, могут приводить к деградации их дозиметрических и спектрометрических свойств. Показано, что такого рода радиационные дефекты являются термически нестабильными. Изотермический отжиг при температуре $350 \leq T \leq 400 \mathrm{~K}$ приводит к диссоциации глубоких радиационных дефектов $\left(E_{3 a}, E_{4 a}\right)$ и восстановлению исходных свойств монокристаллов. Энергия активации отжига, определенная из зависимости концентрации носителей от времени отжига $p(t)$, составляет $E_{D} \approx 0.75$ эВ.

\section{Список литературы}

[1] R.N. Redus, A.C. Huber, J.A. Pantazis. Nucl. Instrum. Meth. A, 458, 214 (2001).

[2] A.V. Rybka, S.A. Leonov, I.M. Prokhorets, A.S. Abuzov, L.N. Davydov, V.E. Kutny, M.S. Rowland, C.F. Smith. Nucl. Instrum. Meth. A, 458, 248 (2001).

[3] H. Miyamaru, T. Iida, A. Takahashi. J. Nucl. Sci. Technol., 36, 54 (1999).
[4] A. Cavallini, B. Fraboni, W. Dusi, M. Zanarini. J. Appl. Phys., 94, 3135 (2003).

[5] P. Hoschl, P. Moravec, J. Franc, E. Belas, R. Grill. Nucl. Instrum. Meth. Phys. Res. A, 322, 371 (1992).

[6] T.E. Schlesinger, R.B. James. Semiconductors for Room Temperature Nuclear Detector Applications [Semiconductors and Semimetals (San Diego, CA: Academic Press, 1995) v. 43].

[7] M. Hage-Ali, P. Siffert. Nucl. Instrum. Meth. Phys. Res. A, 322, 313 (1992).

[8] Z. Huang, E. Eissler, C. Wie. Nucl. Instrum. Meth. Phys. Res. B, 100, 507 (1995).

[9] С.В. Пляцко, Л.В. Рашковецкий. ФТП, 40 (3), 287 (2006).

[10] S.V. Plyatsko, T.L. Petrenko, F.F. Sizov. Infr. Phys. Technol., 52, 57 (2009).

[11] С.В. Пляцко, Л.В. Рашковецкий. ФТП, 47, 890 (2013).

[12] Ж. Бургуэн, М. Лано. Точечные дефекты в полупроводниках (М., Мир, 1985).

[13] D.P. Halliday, M.D.G. Potter, J.T. Mullins, A.W. Brinkman. J. Cryst. Growth, 220, 30 (2000).

[14] S. Seto, K. Suzuki, V.N. Abastillas, K. Inabe. J. Cryst. Growth, 214/215, 974 (2000).

[15] D.M. Hofmann, W. Stadler, K. Oettinger, B.K. Meyer, P. Omling, M. Salk, K.W. Benz, E. Weigel, G. Muller-Vogt. Mater. Sci. Eng. B, 16, 128 (1993).

[16] J.R. Haynes. Phys. Rev. Lett., 4, 361 (1960).

Редактор Г.А. Оганесян

\section{Deep centers of radiation defects in CdZnTe monocrystalls created by fast neutron flux}

\section{S.V. Plyatsko, L.V. Rashkovetskyi}

Lashkarev Institute of Semiconductor Physics, National Academy of Sciences of Ukraine, 03028 Kyiv, Ukraine

Abstract The influence of fast neutron flux $(\Phi=$ $=10^{14}-10^{15} \mathrm{~cm}^{-2}$ ) on electrophysical and photoluminescence properties of $p$-CdZnTe monocrystalls was investigated. The isothermal annealing has been carried out at temperatures $T=400-500 \mathrm{~K}$ and the energy of activation of dissociation of radiation defects was determined and was equal to $E_{D} \approx 0.75 \mathrm{eV}$. 